\title{
Measuring subjective well-being from a multidimensional and temporal perspective: Italian adaptation of the I COPPE scale
}

Salvatore Di Martino ${ }^{* *}$ D, Immacolata Di Napoli ${ }^{2}$, Ciro Esposito ${ }^{2}$, Isaac Prilleltensky ${ }^{3}$ and Caterina Arcidiacono ${ }^{2}$

\begin{abstract}
Background: The objective of this study is to present the psychometric and cultural adaptation of the I COPPE scale to the Italian context. The original 21-item I COPPE was developed by Isaac Prilleltensky and colleagues to integrate a multidimensional and temporal perspective into the quantitative assessment of people's subjective well-being. The scale comprises seven domains (Overall, Interpersonal, Community, Occupation, Psychological, Physical, and Economic well-being), which tap into past, present, and future self-appraisals of well-being.
\end{abstract}

Methods: The Italian adapted version of the I COPPE scale underwent translation and backtranslation procedure. After a pilot study was conducted on a local sample of 683 university students, a national sample of 2432 Italian citizens responded to the final translated version of the I COPPE scale, 772 of whom re-completed the same survey after a period of four months. Respondents from both waves of the national sample were recruited partly through on-line social networks (i.e. Facebook, Twitter, and SurveyMonkey) and partly by university students who had been trained in Computer-Assisted Survey Information Collection.

Results: Data were first screened for non-valid cases and tested for multivariate normality and missing data. The correlation matrix revealed highly significant correlation values, ranging from medium to high for nearly all congeneric variables of the I COPPE scale. Results from a series of nested and non-nested model comparisons supported the 7-factor correlated-traits model originally hypothesised, with factor loadings and inter-item reliability ranging from medium to high. In addition, they revealed that the I COPPE scale has strong internal reliability, with composite reliability always higher than .7, satisfactory construct validity, with average variance extracted nearly always higher than .5, and and full strict invariance across time.

Conclusions: The Italian adaptation of the I COPPE scale presents appropriate psychometric properties in terms of both validity and reliability, and therefore can be applied to the Italian context. Some limitation and recommendations for future studies are discussed.

Keywords: Multidimensional well-being, Time perspective, Confirmatory factor analysis, Construct validity, Composite reliability, Measurement invariance

\footnotetext{
* Correspondence: S.Di-Martino@leedsbeckett.ac.uk

${ }^{1}$ School of Health and Community Studies, Leeds Beckett University, Portland

Building, Room 519, Leeds LS1 3HE, UK

Full list of author information is available at the end of the article
}

(c) The Author(s). 2018 Open Access This article is distributed under the terms of the Creative Commons Attribution 4.0 International License (http://creativecommons.org/licenses/by/4.0/), which permits unrestricted use, distribution, and reproduction in any medium, provided you give appropriate credit to the original author(s) and the source, provide a link to the Creative Commons license, and indicate if changes were made. The Creative Commons Public Domain Dedication waiver (http://creativecommons.org/publicdomain/zero/1.0/) applies to the data made available in this article, unless otherwise stated. 


\section{Background}

For centuries subjective well-being has been the object of philosophical investigation and in recent decades has started to attract the interest of several other disciplines, such as psychology and economics [1]. However, some have pointed out how hard - and sometimes even counterproductive - it is to find an overarching definition of this construct [2]. The scientific literature, in fact, has long acknowledged that subjective well-being has a complex and variegated nature [3, 4]. This understanding stems from at least four main theoretical traditions, that is: Hedonic, Eudaimonic, Quality of Life, and Wellness $[5,6]$. Each of them offers a different and specific perspective into the meaning of subjective well-being.

According to the hedonic approach, well-being is a general experience of pleasure, satisfaction with life, absence of negative affect, and presence of positive affect [7-9]. Eudaimonic well-being entails the achievement of an optimal psychological functioning through the personal development of autonomy, environmental mastery, personal growth, positive relations with others, purpose in life, and self-acceptance $[10,11]$. The quality of life (QoL) approach measures the impact of a broad range of people's life domains, such as physical health, psychological state, level of independence, social relationships, and relationship to salient features of the environment $[12,13]$. Similarly, the wellness approach considers well-being as a holistic construct including multiple areas of health and functioning, such as physical and spiritual health as well as possessing an integrated personality [14].

Based on one or a combination of the above approaches, the literature has produced a substantial number of quantitative subjective well-being instruments [14-17]. Among them, we would like to introduce to the Italian context one recently developed by Prilleltensky and colleagues [18], for the assessment of Overall, Interpersonal, Community, Occupation, Physical, Psychological, and Economic well-being (I COPPE). The theory behind the I COPPE scale posits that well-being is " $a$ positive state of affairs, brought about by the simultaneous and balanced satisfaction of diverse objective and subjective needs of individuals, relationships, organizations, and communities" ([18], p. 2). This definition acknowledges that well-being is both a multilevel and multidimensional construct. It is multilevel because it emphasizes an ecological and systemic perspective that goes beyond the individual to encompass different levels of analysis. It is also multidimensional because it covers different aspects of people's lives, which are all relevant to explain their state of well-being [6]. Moreover, this perspective brings together elements from the hedonic (satisfaction of needs), eudemonic (life fulfilment) and wellness/Quality of Life perspective (health and functioning) into an integrated tool for the subjective well- being assessment. In fact, the I COPPE scale is composed of a total of 21 items tapping into 7 correlated well-being domains (i.e. 3 items per each domain). The following is a descriptive list and operational description of the 7 domains comprising the I COPPE scale: a) Overall Well-being: positive state of affairs, as perceived by individual respondents; b) Interpersonal Well-being: satisfaction with the quality of relationships with important people such as family, friends, and colleagues c) Community Well-being: satisfaction with one's community; d) Occupational Well-being: satisfaction with one's job, vocation, or avocation; e) Physical Well-being: state of satisfaction with one's overall health and wellness; f) Psychological Well-being: satisfaction with one's emotional life; and g) Economic Well-being: satisfaction with one's financial situation.

Beyond its multidimensional nature, a further characteristic of the I COPPE scale is the capacity to integrate a time perspective. The study of time in the evaluation of people's well-being represents a relevant area of enquiry $[19,20]$. For instance, Zimbardo and Boyd [21] have shown that a certain disposition towards time (i.e. past negative, past positive, present hedonic, present fatalistic and future) is strongly linked to different levels of well-being. In particular, past positive, present hedonic, and future predispositions are positively correlated to the experience of subjective well-being [22]. In addition, people who possess balanced time skills, that is, the capacity to shift across different time perspectives depending on the circumstances [21, 23], are generally happier than those who tend to rely only on one specific attitude to time [24].

However, time is also an element extensively overlooked - and often altogether absent - in most well-being instruments. The I COPPE scale represents a fortunate exception since it places each domain of wellbeing on a temporal continuum spanning from past to present and future. Its capacity to embed a temporal perspective in the assessment of subjective well-being lends this tool to several applications, among which are:

i) Comparing levels of well-being in instances where it would be impractical to retrieve information on people's past and/or track them down in the future;

ii) Exploring the impact of life-changing events (i.e. traumas, life transitions, and turning points) and how these experiences are likely to shape people's perception of their past, present, and future wellbeing.

Based on the advantages that the I COPPE scale offers, we deemed a good opportunity to introduce it to the Italian scholarship, which already boasts a well-established tradition of well-being research along with a number of 
already validated well-being instruments [25]. However, the latter are all limited to a selected number of life domains such as a) emotional, social, and psychological wellbeing, in Keyes' Mental Health Continum Short Form [26]; b) autonomy, personal growth, environmental mastery, purpose in life, positive relations, and selfacceptance, in Ryff's Social Scale of Well-being [27], and c) global well-being, in Diener's Satisfaction with Life Scale (SWLS) [28]. Moreover, none of the above-mentioned instruments embeds a time perspective.

Conversely, the I COPPE scale offers the advantage of a wider range of well-being domains along with a time continuum. Therefore, we believe it represents a good addition to Italian well-being literature as well as an opportunity to bridge the gap left by the previous scales.

This tool has already been employed as part of a study comparing Italian and Serbian university students, on which occasion it showed a good level of adaptability to the Italian culture [29]. However, the authors proposed only an alternative shortened version of the I COPPE scale. This study, on the other hand, shows the results of a rigorous process of validation of its full version. In the following pages, we will report the results of construct validity, reliability, as well as model comparisons and time invariance of the Italian version of the I COPPE scale.

\section{Method}

\section{Translation and back translation}

The 21-item Italian version of the I COPPE scale underwent translation and back-translation [30] to establish equivalence of meaning between the source language (i. e. American English) and the target language (i.e. Italian). Following the COSMIN's guideline [31] on cross-cultural validity, we selected four people to form the translation team (two in charge of the translations, one who oversaw the process, and the original developer). The team was composed of experienced researchers, who are proficient in both languages and who worked independently from each other during the translation and back-translation phase. Only minimal discrepancies between the two transated versions of the I COPPE were found. These were in all instances successfully resolved by the translation team.

Following Douglas and Craig's guidelines [32], we conducted a pilot study on a local sample of university students. The pilot version of the Italian adapted I COPPE scale was completed by a local stratified random sample of 683 university students (mean age $=22.633$, SD (2.827), women $=60.1 \%$, men $=38.7 \%$ ), who were at the time enrolled on Bachelor's (73.9\%) and Master's degree (26.06\%) courses at the University of Naples Federico II.

Although the scale showed psychometric good fit, $x^{2}$ $(135)=219.231, p<.001, \mathrm{CFI}=.983$, TLI $=.974$, RMSEA
$=.031,90 \%$ CI [.023 .038], SRMR $=.046$, the qualitative oral feedback collected from the respondents suggested that we improve the readability of the questionnaire. On this account, a second phase of teamwork developed a new adaptation of the instrument, with streamlined and clearer language and instructions (e.g. the common stem question was repeated only for the first item introducing each well-being domain). This new version was presented to a small sample of informed non-specialists and further reviewed by the research team, generating a high agreement over its face validity. Therefore, we employed this as the final adapted version of the Italian I COPPE scale (available in Additional file 1).

\section{Samples and procedures}

Following the above-mentioned changes, the final version of the I COPPE scale was completed by a national sample of 2432 Italian citizens (North $=37.1 \%$, Centre $29.9 \%$, and South \& Islands $=32.8 \%)$. The data collected were first screened for non-Italian residents, people aged under 18 (mean age $=30.528, S D=11.759$ ), and those who did not legally consent to share their sensitive personal data or did not sign the electronic consent. The final sample consisted of 2017 respondents.

The sampling strategy made use of both convenience sampling and snowball sampling. 1291 respondents (64\% of the sample) had been recruited partly trough the private contact network of the research team and in larger part through telephone interviews, which were conducted by 110 undergraduate trained students. The remaining 726 participants (36\% of the sample) were recruited through online social networks (e.g. Facebook, Twitter). All participants were instructed to complete the Italian ICOPPE scale through the online SurveyMonkey platform, where they could also find information about the research and instructions on how to fill out the questionnaire.

The demographic characteristics of the sample are described in Table 1.

The undergraduate students that collaborated in the recruitment phase, were appropriately trained during a 7-day workshop in telephone interview condution and Computer-Assisted Survey Information Collection (CASIC) [33, 34]. During the recruitment phase, they used Prepared Data Entry (PDE) to direct the respondents to the online survey. They resorted to Computer Assisted Telephone Interview (CATI) only whenever necessary, to help those in need of assistance (e.g. no access to the Internet or lack of IT skills, the visuallyimpaired, and some older people) to fill out the online questionnaire.

Data were transferred to Mplus 7.0 and checked for possible biases due to the employment of the two methods of data collection. Wald test showed that there was no statistically significant difference at the chosen $5 \%$ alpha level 
Table 1 Particiant Demographics

\begin{tabular}{|c|c|c|c|c|c|}
\hline \multicolumn{2}{|l|}{$\begin{array}{l}\text { Local Sample } \\
(n=683)\end{array}$} & \multicolumn{2}{|l|}{$\begin{array}{l}\text { National Sample } \\
(n=2017)\end{array}$} & \multicolumn{2}{|l|}{$\begin{array}{l}\text { National Sample } \\
\text { 2nd Wave } \\
(n=696)\end{array}$} \\
\hline Variable & Mean(SD) & Variable & Mean(SD) & Variable & Mean(SD)/ \\
\hline Age & 22.633 (2.827) & Age & 30.528 (11.759) & Age & $\begin{array}{l}29.412 \\
(9.876)\end{array}$ \\
\hline Variable & Frequency in \% & & Frequency in \% & Variable & Frequency in \% \\
\hline Gender & & Gender & & Gender & \\
\hline Male & 38.799 & Male & 40.059 & Male & 33.908 \\
\hline Female & 60.175 & Female & 59.692 & Female & 66.091 \\
\hline Other & / & Other & 0.148 & Other & / \\
\hline Degree Progran & & Territory & & Territory & \\
\hline BSc Degree & 73.932 & North-west & 24.163 & North-west & 20.588 \\
\hline MSc Degree & 26.067 & North-east & 13.030 & North-east & 6.029 \\
\hline Faculty & & Centre & 29.955 & Centre & 28.823 \\
\hline Psychology & 18.448 & South & 26.010 & South & 37.500 \\
\hline Law & 15.373 & Islands & 6.839 & Islands & 7.058 \\
\hline Biology & 14.348 & Civil Status & & Civil Status & \\
\hline Politics & 15.226 & Single & 34.754 & Single & 34.054 \\
\hline Engineering & 15.666 & With partner & 41.249 & With partner & 46.320 \\
\hline Medicine & 14.787 & Married & 20.079 & Married & 16.305 \\
\hline Other & 6.149 & Separated & 1.388 & Separated & 1.443 \\
\hline Curriculum & & Divorced & 0.991 & Divorced & 1.010 \\
\hline Humanities & 51.830 & Widowed & 0.446 & Widowed & .432 \\
\hline \multirow[t]{21}{*}{ Sciences } & 48.169 & Other & 1.090 & Other & .432 \\
\hline & & Education & & Education & \\
\hline & & Primary School & .594 & Primary School & .431 \\
\hline & & Middle School & 8.279 & Middle School & 4.885 \\
\hline & & High School & 51.313 & High School & 42.959 \\
\hline & & Univ. Degree & 29.846 & Univ. degree & 40.660 \\
\hline & & $\begin{array}{l}\text { PhD/Doctorate } \\
\text { Specialization }\end{array}$ & 8.824 & $\begin{array}{l}\text { PhD/Doctorate } \\
\text { Specialization }\end{array}$ & 10.632 \\
\hline & & Other & 1.140 & Other & .431 \\
\hline & & Occupation (Sector) & & Occupation (Sector) & \\
\hline & & $\begin{array}{l}\text { Managerial/ } \\
\text { Professional }\end{array}$ & 11.695 & $\begin{array}{l}\text { Managerial/ } \\
\text { Professional }\end{array}$ & 12.835 \\
\hline & & Employee & 26.251 & Employee & 23.582 \\
\hline & & Secondary sector & .919 & Secondary sector & .298 \\
\hline & & Third sector & 7.201 & Third sector & 7.462 \\
\hline & & Student & 39.968 & Student & 41.940 \\
\hline & & Other & 14.964 & Other & 13.880 \\
\hline & & Occupation (Status) & & Occupation (Status) & \\
\hline & & Unemployed & 25.582 & Unemployed & 29.106 \\
\hline & & Full-time & 35.944 & Full-time & 35.158 \\
\hline & & Part-time & 11.601 & Part-time & 12.103 \\
\hline & & Retired & 1.735 & Retired & 1.152 \\
\hline & & Other & 24.838 & Other & 22.478 \\
\hline
\end{tabular}


between participants contacted by students and those recruited exclusively through online social networks, $\mathrm{W}_{(21)}$ $=24.551, p=.267$.

A total of 1443 respondents from the national sample ( $71.5 \%$ of the sample), had given their availability to be re-contacted in the future for a second administration of the I COPPE scale.

The second wave was launched 4 months after the end of the first wave, through the previously employed online social networks along with email invites sent via the SurveyMonkey platform. ${ }^{1}$ After 322 individuals had answered our survey, a new group of 17 trained undergraduate students re-contacted the rest of the participants, recruiting a further 450 people. Of these, 76 were excluded due to lack of information necessary to match them to their previous data. The final sample comprises a total of 696 respondents. Once more, Wald test shows no statistically significant difference between participants contacted by the students and those who answered our mail invites, $\mathrm{W}_{(21)}=32.132, p=.056$. At the end of each wave, we awarded a raffle prize to a randomly selected respondent. The prizes consisted of $€ 100$ and $€ 200$ Amazon vouchers for the first and second wave respectively, which was intended as a way of thanking the respondents for their participation.

\section{Results}

\section{Data analysis}

Based on the results of the original validation, we used Confirmatory Factor Analysis as implemented in Mplus 7. 0 , to assess the applicability of the I COPPE scale to the Italian context. ${ }^{2}$ The correlation matrix (see Additional file 2) reveals that all the manifest variables used for the I COPPE scale are significantly correlated at the $1 \%$ alpha level. In addition, all congeneric variables show medium to high correlations, except for OV_WB_PA and OV_WB_FU, $r=.274, p=.001$ (see Additional file 2). Mardia's test [35] revealed a clear violation of multivariate normality for both skewness $(\mathrm{M}=5.386, S D=0.186, p<.001)$ and kurtosis $(\mathrm{M}=482.585, S D=1.415, p<.001)$. To address this issue, Maximum Likelihood Robust (MLR) was chosen as main estimator. ${ }^{3}$

To assess model fit, we followed $\mathrm{Hu}$ and Bentler's guidelines [36] according to which the Comparative Fit Index (CFI) and Tucker-Lewis Index (TLI) should be > .95, the Root Mean Square Error of Approximation (RMSEA) $<.05$, and the Standardized Root Mean Square Residual $($ SRMR $)<.08$. In addition to these, the Chisquare value should not be significant at the $5 \%$ alpha level. However, the sensitivity of this test to sample size has been highlighted on several occasions [37, 38] and since the samples recruited in this study are all relatively large, we will ignore its statistical significance.
Missing values were in all instances treated with listwise deletion, with a relatively small loss of cases in all instances. Nonetheless, power analysis based on the RMSEA test of close fit shows that at the 5\% alpha level, with 118 degrees of freedom, ${ }^{4}$ the minimum sample size to reach a power of .8 is 117.968 . This shows that the main analyses we carried out on the Italia I COPPE scale have enough power to confidently avoiding making a Type II error.

As in the original validation of the I COPPE, we allowed residual errors to correlate between manifest variables that shared an item stem, given the hypothesised method effect by time period referenced [39]. Being that this case is a Nonstandard Confirmatory Factor Analysis Model with correlated errors, we applied the rules suggested by Kenny, Kashy, and Bolger ([40], p. 253-254) for identifying our model, that is:

a) each factor has at least three indicators whose errors are uncorrelated with each other,

b) for every pair of constructs there are at least two indicators, one from each construct, that do not have correlated measurement error between them, and

c) for every indicator, there must be at least one other indicator (not necessarily of the same construct) with which it does not share correlated measurement error.

Since all the above conditions are satisfied (see Fig. 1 and Additional file 2), we can consider the proposed Nonstandard Confirmatory Factor Analysis Model as identified.

\section{Results}

The proposed 7-factor correlated-traits model provides a very good fit to the data, $\chi^{2}{ }_{(118)}=155.413, p=.011$, CFI $=.997, \mathrm{TLI}=.995, \mathrm{RMSEA}=.013,90 \%$ CI [.006 .018], $\mathrm{SRMR}=.024$, therefore we can accept the null hypothesis that the model's implied variance-covariance matrix $[\Sigma(\theta)]$ and the model's covariance matrix $[\Sigma]$ are not significantly different.

Figure 1 shows that nearly all congeneric variables have both significant and high factor loading associated to their corresponding latent variable. However, it is also worthy of notice that all the items of past well-being have generally lower standardised factor loadings $(\lambda)$ and inter-item reliability $\left(R^{2}\right)$ than their congeneric variables. Among them, two manifest variables show the lowest values, namely OV_WB_PA, $\lambda^{0}=.455, \mathrm{SE}=.022,95 \% \mathrm{CI}$ $[.412, .499], \mathrm{R}^{2}=.207$ and PS_WB_PA, $\lambda^{0}=.449, \mathrm{SE}$ $=.025,95 \%$ CI [.401, .498], $\mathrm{R}^{2}=.202$.

Although some suggest that standardized loading estimates should be ideally $\geq .5$ for CFA [41], none of them 


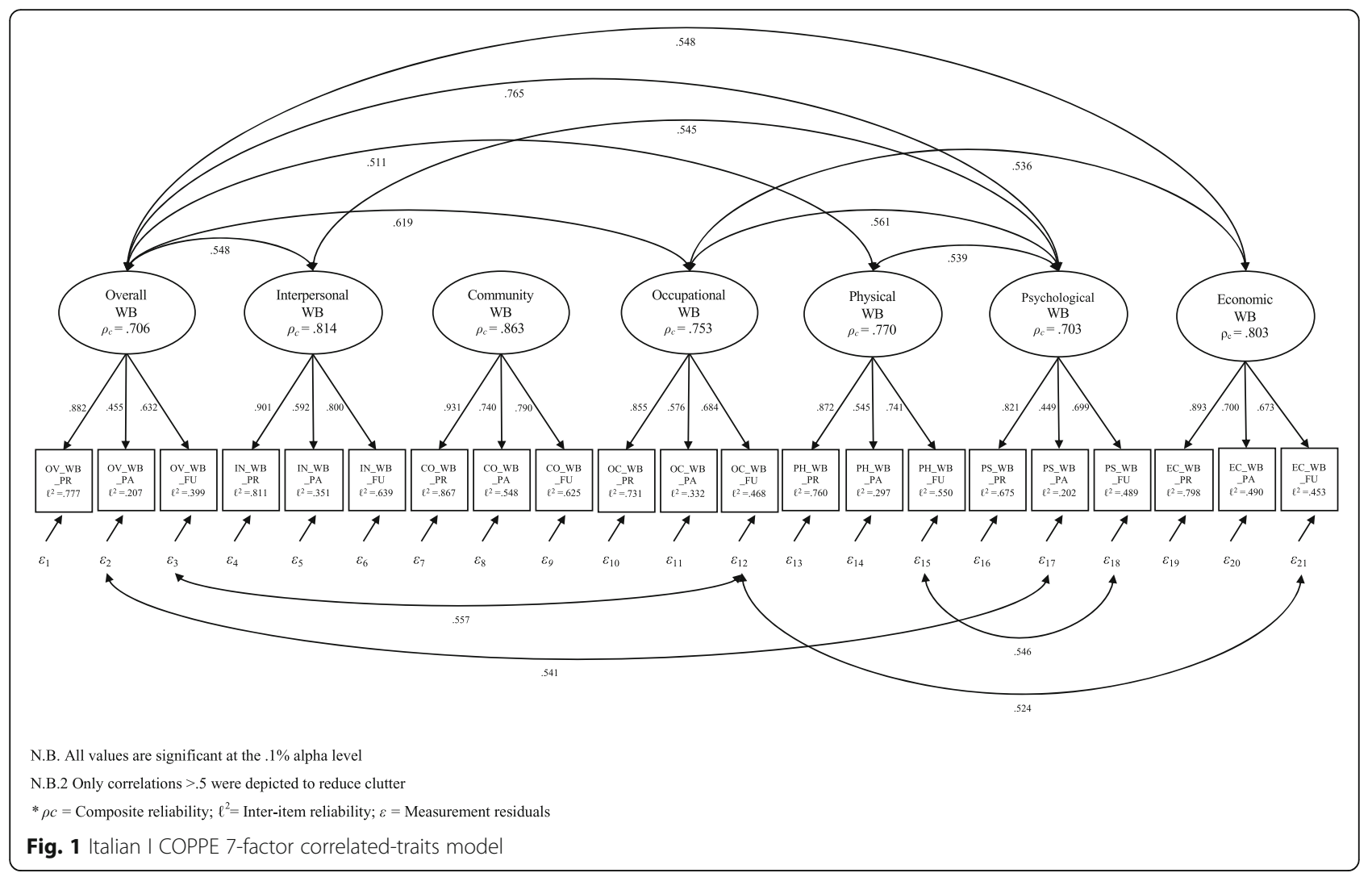

is small enough (i.e. <.40) not to be considered a "salient" factor loading [42].

\section{Model comparisons}

Some studies have demonstrated that alternative structures of the I COPPE scale such as the One-Factor [29] and Bi-Factor [43] solution can better express the variability of subjective well-being in different cultural contexts. On this account, we decided to compare the 7factor correlated-traits model proposed as the original I COPPE scale (Model A) to a series of alternative nested models (see Table 2) to test which one of these would be the most appropriate to apply to the Italian context.

Given the presence of multivariate non-normality, MLR was used as an estimator in all cases. As such, model comparisons were based on the scaled Chi-square difference statistic [44].

In the Second Order solution (Model B), the 7 factors measured in Model A were additionally constrained to load onto a higher order well-being factor. In the MTMM solution (Model C), in addition to Model A, we constrained all the items of the past, present, and future to load on a Past, Present, and Future trait factor respectively in a correlated trait-correlated method model (CTCM). The Bi-Factor solution (Model D) includes a general factor that is orthogonal to the 7 specific factors proposed in Model A. Compared to the previous solutions, Model A is nested within Model D, therefore a significant Chi-square difference statistic would favour the Bi-Factor solution and vice versa. Consistent with what was found by Myers and colleagues [45], the manifest variables of OV_WB loaded significantly only onto the general factor. Lastly, in the One Factor solution (Model E) all the 21 items comprising the I COPPE were allowed to load onto only one general well-being factor. Since Model E is not nested within Model A, it was not possible to compare them through the scaled Chi-square difference statistic. However, the Akaike and (AIC) and Bayesian (BIC) indices displayed in Table 2 - which are useful to compare non-nested models - show that Model A yields a better fit to the data than Model E. In addition, both models are nested within Model D, therefore we could make an indirect comparison, first between Model E and Model D, which favours model D, and then between Model A and Model D, which in turn favours Model A.

Since none of the alternative models proposed provides a better fit to the data than the comparative model (see Table 2), we can conclude that the multidimensional solution with 7 intercorrelated well-being factors is the best fitting model to describe the Italian adaptation of the I COPPE scale. 
Table 2 Model Comparisons between the 7-factor correlated-traits model and alternative models

\begin{tabular}{|c|c|c|c|c|c|}
\hline Model/Indices & $\begin{array}{l}\text { A } \\
7 \text { Factors }\end{array}$ & $\begin{array}{l}\text { B } \\
\text { 2nd Order }\end{array}$ & $\begin{array}{l}\text { C } \\
\text { Multi-Trait } \\
\text { Multi-Method }^{\text {b }}\end{array}$ & $\begin{array}{l}\text { D } \\
\text { Bi-Factor }\end{array}$ & $\begin{array}{l}\text { E } \\
\text { One Factor }\end{array}$ \\
\hline$M L R x^{2}$ & 155.413 & 255.003 & 344.343 & 149.167 & 345.788 \\
\hline$x^{2} d f$ & 118 & 132 & 146 & 108 & 121 \\
\hline$x^{2} p$ & .011 & $<.001$ & $<.001$ & .0054 & $<.001$ \\
\hline CFI & .997 & .991 & .986 & .997 & .984 \\
\hline TLI & .995 & .986 & .979 & .994 & .972 \\
\hline $\begin{array}{l}\text { RMSEA } \\
(90 \% \text { CI) }\end{array}$ & $\begin{array}{l}.013 \\
(.006 .018)\end{array}$ & $\begin{array}{l}.022 \\
(.018 .026)\end{array}$ & $\begin{array}{l}.026 \\
(.023 .030)\end{array}$ & $\begin{array}{l}.014 \\
(.008 .019)\end{array}$ & $\begin{array}{l}.031 \\
(.027 .035)\end{array}$ \\
\hline SRMR & .024 & .032 & .025 & .022 & .028 \\
\hline Akaike (AIC) & $147,929.900$ & $148,049.431$ & $147,888.189$ & $147,938.931$ & $148,205.576$ \\
\hline Bayesian (BIC) & $148,678.395$ & $148,719.725$ & $148,480.121$ & $148,743.285$ & $148,937.314$ \\
\hline Model Comparison & / & B Versus A & C Versus A & A Versus D & E Versus D \\
\hline$\Delta M L R x^{2 a}$ & / & 95.685 & 179.258 & 6.882 & 175.441 \\
\hline$\Delta \mathrm{df}$ & / & 14 & 28 & 10 & 13 \\
\hline$\Delta p$ & / & $<.001$ & $<.001$ & .737 & $<.001$ \\
\hline
\end{tabular}

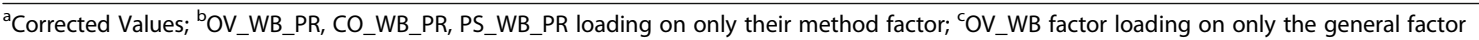

\section{Reliability and construct validity}

To test for the internal reliability of the Italian I COPPE scale, we used Composite Reliability (CR), which is known to perform better than the most commonly-used Cronbach alpha, given the multitude of cases where the condition of tau-equivalence cannot be met [46]. Values of $\rho_{c}>.6$ are considered desirable, and above .7 are indicative of a high level of CR. Table 3 shows that $\rho_{c}$ ranges from a minimum of .703 to a maximum of .863 , indicating a good level of reliability per each factor of the I COPPE scale.

Evidence of the I COPPE's construct validity has been so far ested through Campbell and Fiske's [47] Multitrait-Multimethod Matrix (MTMM) (see [18, 43]). However, the latter has been strongly brought into question, above of all for leaving to the researcher's interpretation the maginitude of the correlations within the MTMM matrix [48]. For this reason, we opted for another widely used alternative, namely the Fornell and Larcker' method (1981), which offers clearer guidelines and cut-off points to assess convergent and discriminant validity (see [41]). Indeed, according to Fornell- Larcker, convergent validity can be assessed through Average Variance Extracted (AVE), which measures the total amount of variance of a construct compared to the variance due to measurement error. According to Fornell and Larcker, values of $\rho_{\bar{\nu}}$ above .5 are index of desirable AVE. In addition, AVE can also be used to test for discriminant validity. In this case, AVE should be higher than Maximum Shared Variance (MSV), and Average Shared Variance (ASV). As we can see from Table 3, all the latent variables of the I COPPE scale meet the above criteria, except for OV_WB $\left(\mathrm{MSV}=.585>\rho_{\bar{v}}=.461\right)$ and PS_WB (MSV $\left.=.585>\rho_{\bar{v}}=.455\right)$. This is probably due to the high correlation between these two factors $\Psi_{\left(\mathrm{OV} \_ \text {WB, PS_WB }\right)}=.765$ (see Table 3 and Fig. 1), the low parameter estimates for OV_WB_PA, $\lambda^{0}=.455, p<.001$, $95 \%$ CI $[.412, .499]$ and PS_WB_PA, $\lambda^{0}=.449, p<.001$, 95\% CI [.401, 498], and the significant high zero-order correlation between their error terms, $\boldsymbol{\varepsilon}=.541, p<.001$. However, this does not pose a serious threat either to their convergent validity (the AVE of both factors is only slightly below the suggested cut-off point ) or to their discriminant validity (the AVE of both factors is always higher than their corresponding ASV).

\section{Time invariance}

In this paragraph we will test the factor equivalence of the I COPPE scale across time (i.e. first and second wave). A commonly adopted practice to test for time invariance in Structural Equation Modeling (SEM) is to start with a group-specific baseline model whereby a partial measurement invariance $[49,50]$ is compared against increasingly constrained SEM models. The first level of invariance tests for equivalence of factor loadings (metric invariance). The next level builds upon metric invariance to further test for equivalence of intercepts (scalar invariance) [51]. Although we also provided evidence of factor invariance and equivalence of indicator residual variances (strict invariance), some argue that the latter might be unduly restrictive and that achieving partial scalar invariance is sufficient in many 
Table 3 Factor Correlations, Reliability and Validity Measures of the Italian I COPPE scale

\begin{tabular}{|c|c|c|c|c|c|c|c|}
\hline $\begin{array}{l}\text { Latent } \\
\text { Variable }\end{array}$ & OV_WB & IN_WB & CO_WB & OC_WB & PH_WB & PS_WB & $E C \_W B$ \\
\hline OV_WB & 1 & & & & & & \\
\hline IN_WB & .548 & 1 & & & & & \\
\hline CO_WB & .439 & .299 & 1 & & & & \\
\hline OC_WB & .619 & .343 & .418 & 1 & & & \\
\hline PH_WB & .511 & .424 & .323 & .389 & 1 & & \\
\hline PS_WB & .765 & .545 & .410 & .561 & .539 & 1 & \\
\hline EC_WB & .498 & .295 & .357 & .536 & .422 & .466 & 1 \\
\hline \multicolumn{8}{|c|}{ Reliability and Validity Measures } \\
\hline $\mathrm{CR}\left(\rho_{c}\right)$ & .706 & .814 & .863 & .753 & .770 & .703 & .803 \\
\hline $\operatorname{AVE}\left(\rho_{\bar{V}}\right)$ & .461 & .601 & .679 & .510 & .535 & .455 & .580 \\
\hline MSV & .585 & .300 & .192 & .383 & .314 & .585 & .287 \\
\hline ASV & .328 & .178 & .150 & .219 & .194 & .312 & .190 \\
\hline
\end{tabular}

N.B. All values are significant at the .1\% alpha level; CR Composite Reliability, AVE Average Variance Extracted, MSV Maximum Squared Shared Variance, ASV Average Shared Square Variance

circumstance $[36,42,52]$. In the light of this, we tested increasingly more restrictive invariances until model fit criteria indicated that the latest set of restrictions is no longer tenable for the data, as recommended by Geiser ([53], p. 101).

To test for the time invariance of the Italian I COPPE scale, we compared the data of 696 respondents, who took part in both the first and second wave of the national sample. The time range of responses that were provided, varies between a minimum of four and a maximum of seven months from the last administration of the scale.

The presence of missing data between the two waves required deleting 118 cases, reducing the final sample to 578 cases. However, this did not significantly alter the power of the test, which still shows a $99 \%$ chance of correctly accepting the null hypothesis that the increasingly restricted models are not significantly different from the configural model.

The configural Model 1.1 provides a satisfactory fit to the data, $\mathrm{X}^{2}{ }_{(629)}=1005.602, \quad p<.001, \mathrm{CFI}=.966$, TLI $=.954, \mathrm{RMSEA}=.032,90 \%$ CI $[.028, .036], \mathrm{SRMR}=.066$.

Comparisons between Model 1.2 versus Model 1.1 (Full Metric Invariance), Model 1.3 versus Model 1.2 (Full Scalar Invariance), and Model 1.4 versus Model 1.3 (Full Strict Invariance), show that factor loadings, intercepts, factor variance, and indicators residual variance, all are equivalent across the two-time points considered (Table 4). Therefore, we can conclude that Full Strict Invariance holds for the Italian I COPPE scale.

\section{Discussion}

The findings presented in this study confirm the original structure identified by Prilleltensky and colleagues [18], since all the alternative models proposed fail to describe the data better than the 7-factor correlated model. This indicates that we can consider the Italian adapted I COPPE scale as a multidimensional instrument tapping into different, yet related, domains of subjective wellbeing.

The value of $\mathrm{CR}$ showed that all the factors of the I COPPE scale have a high level of internal reliability. Furthermore, AVE provided strong evidence of both convergent and discriminant validity, except for Overall Well-being and Psychological Well-being. Although their validity is still partially tenable, we could suggest at least two strategies for future researchers, should they encounter a more severe lack of validity [54]. The first is to delete the items with the largest measurement error variance (i.e. Overall and Psychological Past Well-being). In our case, the model fit remains nearly unaltered, $X^{2}{ }_{(92)}=124.992, p=.012, C F I=.997$, $\mathrm{TLI}=.995, \quad \mathrm{RMSEA}=.013(.007, .019), \quad \mathrm{SRMR}=.022$, with a substantial increase in AVE for both Overall Well-being, $\rho_{\bar{\nu}}=.586$ and Psychological Well-being $\rho_{\bar{v}}=.586$, which are now only slightly smaller than their corresponding Maximum Shared Variance $=.594$. Another strategy would be to collapse the overlapping dimensions into a single factor. Although the resulting model fit is statistically equivalent to the correlated seven-factor model due to the lack of overidentification, the second-order factor shows a very good level of AVE, $\rho_{\bar{v}}=.768$, which is much higher than its corresponding MSV $=.458$.

The test for time invariance shows that the Italian I COPPE scale is consistent in measuring the 7 domains of subjective well-being across time. This is in line with 
Table 4 Italian I COPPE scale Time invariance results (1st and 2nd wave)

\begin{tabular}{|c|c|c|c|c|}
\hline Model/Indices & $\begin{array}{l}1.1 \\
\text { Configural Model }\end{array}$ & $\begin{array}{l}1.2 \text { Full } \\
\text { Metric Invariance }\end{array}$ & $\begin{array}{l}1.3 \text { Full } \\
\text { Scalar } \\
\text { Invariance }\end{array}$ & $\begin{array}{l}\text { 1.4 Full } \\
\text { Strict Invariance }\end{array}$ \\
\hline$M L R x^{2}$ & 1005.602 & 1021.211 & 1040.046 & 1055.228 \\
\hline$x^{2} d f$ & 629 & 643 & 658 & 686 \\
\hline$x^{2} p$ & $<.001$ & $<.001$ & $<.001$ & $<.001$ \\
\hline $\mathrm{CFI}$ & .966 & .966 & .966 & .968 \\
\hline TLI & .954 & .955 & .955 & .960 \\
\hline $\begin{array}{l}\text { RMSEA } \\
(90 \% \mathrm{Cl})\end{array}$ & $\begin{array}{l}.032 \\
(.028 .036)\end{array}$ & $\begin{array}{l}.032 \\
(.028 .036)\end{array}$ & $\begin{array}{l}.032 \\
(.028 .035)\end{array}$ & $\begin{array}{l}.030 \\
(.026 .033)\end{array}$ \\
\hline SRMR & .066 & .067 & .067 & .070 \\
\hline Model Comparison & / & 1.2 Vs 1.1 & 1.3 Vs 1.2 & 1.4 Vs 1.3 \\
\hline$\triangle M L R X^{2 a}$ & l & 17.677 & 14.561 & 24.942 \\
\hline$\Delta \mathrm{df}$ & / & 14 & 15 & 28 \\
\hline$P$ & / & .222 & .483 & .631 \\
\hline
\end{tabular}

${ }^{\text {a }}$ Corrected Values

similar results found in the literature, which suggest that subjective well-being might be a stable psychological trait $[55,56]$, in that it is unlikely to be permanently influenced by the respondent's situational variability such as daily mood, and more likely to be affected by lifechanging events and/or contextual variables $[45,57]$.

Lastly, all the manifest variables used show a strong relation to their corresponding domain of subjective well-being, except for the items of the past. Our findings are consistent with some previous analyses conducted on the I COPPE scale [45], which concluded that "an individual's perceptions of the past, at least in some circumstances, may offer negligible empirical contributions over and above an individual's perceptions of the present and future in the practical assessment of multidimensional well-being" (p. 796).

\section{Limitations}

The generability of the results presented in this paper should be interpreted in the context of some limitations. Although we strived not to pose restrictions to the participation in this study, a high number of respondents who took the online survey had to be contacted through snowball sampling and convenience sampling strategies. In addition, the majority of respondents had a level of IT literacy and access to a computer, the internet, and social networks and even the small percentage who were assisted through CATI still owned a telephone. This poses some limitations to the generalisability of the results to the whole of the Italian population. Future uses of the I COPPE scale with random national samples could offer further evidence to the results we obtained.

Another limitation pertains to the number of wellbeing domains composing the I COPPE scale. In the original validation study, Prilleltensky and colleagues [18] identified a possible limitation of the I COPPE scale in "the possibility that other potentially important factors... also contribute meaningfully to well-being" (p. 212-213). In that regard, Linton and colleagues [14] recently conducted a systematic review on self-reported measures of well-being, in which they showed that the I COPPE scale encompasses six of the seven domains they found to be core components of subjective well-being, that is: overall well-being, mental well-being, social well-being, physical well-being, spiritual well-being, activities and functioning, and personal circumstances. We suggest that a future revised version of the I COPPE scale integrates Spiritual Well-being, the only relevant domain currently missing. This would contribute to placing this tool among the most comprehensive quantitative instruments for the assessment of subjective well-being.

We should also bear in mind that the I COPPE scale was designed primarily to measure subjective well-being at the individual level of analysis. As such, it should always be used in combination with other objective indicators as well as further methods to assess well-being at the community and social level [58-60].

Lastly, the I COPPE scale remains a quantitative instrument for the general assessment of people's multiple domains of subjective well-being. Therefore, its use should be discouraged - or at least readapted - in contexts where specific life circumstances play a strong role in people's assessment of their own subjective well-being.

\section{Conclusions}

Our results provide empirical evidence in support of the thesis that the Italian adaptation of the I COPPE scale is a valid and reliable instrument. Indeed, evidence of good construct validity (i.e. convergent and discriminant validity), coupled with strong internal reliability and time 
invariance support our thesis that the I COPPE scale can be adapted to the Italian context. However, given the nature of the sampling strategies we used, we still advice caution in generalising the results presented here to the whole of the Italian population.

The main strength of this tool lies in its multidimensional nature, which encompasses nearly all the key components of subjective well-being currently identified in the literature. In addition, the I COPPE scale is almost unique in incorporating time variability, showing how people's perception of their subjective well-being is likely to change from past to present and future.

Therefore, we believe that the evidence offered in this study constitutes an opportunity for Italian scholars, clinicians, activists, and practitioners to further investigate the nature of subjective well-being from a multidimensional and temporal perspective. In that regard, this tool can contribute to expand those research fields such as community psychology, public health, and health economics, only to name a few, that are currently investiganting both in Italy and abroad - the intrinsic relationship between well-being and the resources provided by the environment. The flexibility in its use at the individual, organizational, and community level, makes the I COPPE scale a window onto the contextual nature of subjective well-being while acknowledging its strong link with multiple domains of life and temporal variability.

\section{Endnotes}

${ }^{1}$ In the absence of clear guidelines in the literature, we opted for a time interval we believed to be sufficiently wide to avoid recollection biases.

${ }^{2}$ All the analyses and results described in this and the next paragraphs refer to the national sample

${ }^{3}$ All the congeneric variables composing the I COPPE are measured on a ratio scale ranging from 0 to 10 .

${ }^{4}$ These figures pertain to the 7 -factors correlated-traits model, which was used as main explanatory model.

\section{Additional files}

Additional file 1: । COPPE Scale Italian Adaptation. (DOCX $337 \mathrm{~kb}$ )

Additional file 2: Manifest variables Correlation Matrix. (DOCX 18 kb)

\section{Abbreviations}

ASV: Average Shared Variance; AVE: Average Variance Extracted; CFI: Comparative Fit Index; Cl: Confidence Interval; CO_WB: Community Wellbeing; CR: Composite Reliability; EC_WB: Economic Well-being; FU: Future; IN_WB: Interpersonal Well-being; MLR: Maximum Likelihood Robust; MSV: Maximum Shared Variance; OC: Occupational Well-being; OV_WB: Overall Well-being; PA: Past; PH_WB: Physical Well-being; PR: Present; PS_WB: Psychological Well-being; $R^{2}$ : Individual Inter-item reliability; RMSEA: Root Mean Square Error of Approximation; SD: Standard Deviation; SE: Standard error; SEM: Structural Equation Modeling; SRMR: Standardized Root Mean Square Residual; TLI: Tucker-Lewis Index

\section{Acknowledgments}

We heartily thank all the trained students who contributed to the data collection; their cooperation and commitment has been integral to the realization of this work. We also would like to express our gratitude to all the respondents who have so generously provided the necessary data for this research. Lastly, we wish to thank Dr. Nicholas Myers, for the helpful comments he shared about the original version of the I COPPE scale.

\section{Availability of data and materials}

The dataset used and/or analysed during the current study is available from the corresponding author on reasonable request.

\section{Authors' contributions}

SDM and CA conceived and designed the study as well as collaborated in the translation/back-translation of the instrument. SDM and CE oversaw and participated in the acquisition of the data. SDM performed the statistical analyses and contributed to the methodological section. IDN and CE contributed to the background section and literature search. CA and IP provided critical reviews and revisions. All authors read and approved the final manuscript.

\section{Ethics approval and consent to participate}

The study protocol was approved by the ethics committee of the University of Naples Federico II. All respondents gave their informed consent to be included in this study. Permission has been obtained from the developers of the I COPPE scale to translate it into the Italian version.

\section{Competing interests}

The authors declare that they have no competing interests.

\section{Publisher's Note}

Springer Nature remains neutral with regard to jurisdictional claims in published maps and institutional affiliations.

\section{Author details}

${ }^{1}$ School of Health and Community Studies, Leeds Beckett University, Portland Building, Room 519, Leeds LS1 3HE, UK. '² Department of Humanities, University of Naples Federico II, Via Porta di Massa 5, 80133 Naples, IT, Italy. ${ }^{3}$ School of Education and Human Development, University of Miami, Merrick Building 312, 5205 University Drive, Coral Gables, Florida 33146, USA.

Received: 10 July 2017 Accepted: 29 April 2018

Published online: 08 May 2018

\section{References}

1. Stoll L. A short history of wellbeing research. In: McDaid D, Cooper CL, editors. The economics of wellbeing: wellbeing a complete reference guide. Oxford: Wiley; 2014. p. 1-19.

2. Dodge R, Daly AP, Huyton J, Sanders LD. The challenge of defining wellbeing. Int J Well-being. 2012;2(3):222-35.

3. Christopher J. Situating psychological well-being: exploring the cultural roots of its theory and research. J Couns Dev. 1999;77:141-52.

4. Pollard E, Lee P. Child well-being: a systematic review of the literature. Soc Indic Res. 2003;61(1):9-78.

5. Cooke PJ, Melchert TP, Connor K. Measuring well-being: a review of instruments. Couns Psychol. 2016;44(5):730-57.

6. Arcidiacono C, Di Martino S. A critical analysis of happiness and well-being. Where do we stand now, where do we want to go? Happiness and social well-being. Community Psychol Global Perspect. 2016;2(1):6-35.

7. Ryan RM, Deci EL. On happiness and human potentials: a review of research on hedonic and eudaimonic well-being. Annu Rev Psychol. 2001:52(1):141-66.

8. Diener E. Subjective well-being: the science of happiness, and a proposal for a national index. Am Psychol. 2000;55:34-43.

9. Diener E, Lucas R, Schimmack U, Helliwell J. Well-being for public policy. Oxford: OUP; 2009.

10. Ryff C. Happiness is everything, or is it? Explorations on the meaning of psychological well-being. J Pers Soc Pychol. 1989;57:1069-81.

11. Ryff CD, Keyes CLM. The structure of psychological well-being revisited. J Pers Soc Psychol. 1995;69(4):719-27. 
12. Group TW. The World Health Organization quality of life assessment (WHOQOL): development and general psychometric properties. Soc Sci Med. 1998;46(12):1569-85.

13. Frisch MB, Cornell J, Villanueva M, Retzlaff PJ. Clinical validation of the quality of life inventory. A measure of life satisfaction for use in treatment planning and outcome assessment. Psychol Assessment. 1992:4(1):92-101.

14. Linton MJ, Dieppe P, Medina-Lara A, Watson L, Crathorne L. Review of 99 self-report measures for assessing well-being in adults: exploring dimensions of well-being and developments over time. B M J Open. 2016 https://doi.org/10.1136/bmjopen-2015-010641.

15. Dronavalli $M$, Thompson SC. A systematic review of measurement tools of health and well-being for evaluating community-based interventions. J Epidemiol Community Health. 2015;69(8):808-15.

16. Charlemagne-Badal SJ, Lee JW, Butler TL, Fraser GE. Conceptual domains included in wellbeing and life satisfaction instruments: a review. Appl Res Qual Life. 2015;10(2):305-28.

17. Di Martino S, Arcidiacono C, Eiroa-Orosa FJ. Happiness and well-being revisited: including the role of context, justice and values in our understanding of the good life. In: Brown NJL, Lomas T, Eiroa-Orosa FJ, editors. Handbook of critical positive psychology — a synthesis for social change. London: Routledge; 2017. p. 99-116.

18. Prilleltensky I, Dietz S, Prilleltensky O, Myers N, Rubenstein C, Jin Y, McMahon A. Assessing multidimensional well-being: development and validation of the I COPPE scale. J Community Psychol. 2015;43:199-226.

19. Bronfenbrenner U. Interacting Systems in Human Development. Research paradigms: present and future. In: Bolger N, Caspi A, Downey G, Moorehouse $M$, editors. Persons in context: developmental processes. Cambridge: Cambridge University Press; 1988. p. 25-49.

20. Bronfenbrenner U. Ecological models of human development. In: Husten T, Postlethwaite TN, editors. International encyclopedia of education, vol. 3. 2nd ed. New York: Elsevier Science; 1994. p. 1643-7.

21. Zimbardo PG, Boyd JN. Putting time in perspective: a valid, reliable individual-difference metric. J Pers Soc Psychol. 1999;77:1271-88.

22. Zimbardo PG, Keough KA, Boyd JN. Present time perspective as a predicto of risky driving. Pers Individ Dif. 1997;23(6):1007-23.

23. Zimbardo PG, Boniwell I. Balancing one's time perspective in pursuit of optimal functioning. Positive psychology in practice. Hoboken: Wiley; 2004

24. Drake L, Duncan E, Sutherland F, Abernethy C, Henry C. Time perspective and correlates of well-being. Time Soc. 2008;17(1):47-61.

25. Delle Fave A. Dimensions of well-being: research and intervention. Milano: Franco Angeli; 2006

26. Petrillo G, Capone V, Caso D, Keyes CLM. The mental health continuumshort form (MHC-SF) as a measure of well-being in the Italian context. Soc Indic Res. 2015;121(1):291-312.

27. Ruini C, Ottolini F, Rafanelli C, Ryff CD, Fava GA. La validazione italiana delle Psychological Well-being Scales (PWB). Riv Psichiatr. 2003;38(3):117-30.

28. Di Fabio A, Busoni L. (2009). Proprietà psicometriche della versione italiana della Satisfaction With Life Scale (SWLS) con studenti universitari. Counseling. Giornale Italiano di Ricerca e Applicazioni. 2009:2:201-12

29. Lietz F, Piumatti G, Mosso C, Marinkovic J, Bjegovic-Mikanovic V. Testing multidimensional well-being among university community samples in Italy and Serbia. Health Promot Int. 2016. https://doi.org/10.1093/heapro/daw082

30. Brislin RW. Back-translation for cross-cultural research. J Cross-Cult Psychol. 1970;1(3):185-216.

31. Mokkink LB, Terwee CB, Patrick DL, Alonso J, Stratford PW, Knol DL, et al. The COSMIN checklist for assessing the methodological quality of studies on measurement properties of health status measurement instruments: an international Delphi study. Qual Life Res. 2010;19(4):539-49.

32. Douglas SP, Craig CS. Collaborative and iterative translation: an alternative approach to back translation. J Int Mark. 2007;15(1):30-43.

33. Couper MP, Baker RP, Bethlehem J, Clark CZF, Martin J, Nicholls WL, O'Reilly JM. Computer assisted survey information collection. New York: John Wiley \& Sons; 1998.

34. Weeks MF. Computer-assisted survey information collection: a review of CASIC methods and their implications for survey operations. J Off Stat. 1992; 8(4):445-65.

35. Mardia KV. Measures of multivariate skewness and kurtosis with applications. Biometrika. 1970;57(3):519-30.

36. Hu L, Bentler PM. Cutoff criteria for fit indexes in covariance structure analysis: conventional criteria versus new alternatives. Struct Equ Modeling. 1999;6(1):1-55.
37. Fornell C, Larcker DF. Evaluating structural equation models with unobservable variables and measurement error. J Mark Res. 1981;19:39-50.

38. Bentler PM, Bonett DG. Significance tests and goodness of fit in the analysis of covariance structures. Psychol Bull. 1980;88(3):588-606.

39. Saris WE, Aalberts $C$. Different explanations for correlated disturbance terms in MTMM studies. Struct Equ Modeling. 2003;10:193-213.

40. Kenny DA, Kashy D, Bolger N. Data analysis in social psychology. In: Gilbert D, Fiske S, Lindzey G, editors. Handbook of social psychology. 4th ed. New York: McGraw-Hill; 1998. p. 233-65.

41. Hair JFJ, Black WC, Babin BJ, Anderson RE. Multivariate data analysis (7th edition). Pearson Education Limited: Harlow; 2014

42. Brown TA. Confirmatory factor analysis for applied research. New York: Guilford Publications, Incorporated; 2006.

43. Myers ND, Park SE, Lefevor GT, Dietz S, Prilleltensky I, Prado GJ. Measuring multidimensional subjective well-being with the I COPPE scale in a Hispanic sample. Meas Phys Educ Exerc Sci. 2016;20(4):230-43.

44. Satorra A, Bentler PM. A scaled difference chi-square test statistic for moment structure analysis. Psychometrika. 2001;66(4):507-14.

45. Myers ND, Prilleltensky I, Jin Y, Dietz S, Rubenstein CL, Prilleltensky O, McMahon A. Empirical contributions of the past in assessing multidimensional well-being. J Community Psychol. 2014;42(7):789-98.

46. Raykov T. Estimation of composite reliability for congeneric measures. Appl Psychol Meas. 1997;21(2):173-84.

47. Campbell DT, Fiske DW. Convergent and discriminant validation by the multitrait-multimethod matrix. Psychol Bull. 1959;56(2):81.

48. Ferketich SL, Fiqueredo AJ, Knapp TR. Focus on psychometrics. The multitrait-multimethod approach to construct validity. Res Nurs Health. 1991;14(4):315-20.

49. Byrne BM. Structural equation modeling with Mplus: basic concepts, applications, and programming. New York: Taylor \& Francis; 2013

50. Byrne BM, Shavelson RJ, Muthén B. Testing for the equivalence of factor covariance and mean structures: the issue of partial measurement invariance. Psychol Bull. 1989:105(3):456-66.

51. Widaman KF, Reis SP. Exploring the measurement invariance of psychological instruments: applications in the substance use domain. In: Bryant MW, West SG, editors. The science of prevention: methodological advances from alcohol and substance abuse research. Washington, DC: American Psychological Association; 1997. p. 281-324.

52. Marsh HW. Confirmatory factor analysis models of factorial invariance: a multifaceted approach. Struct Equ Modeling. 1994;1 (1):5-34.

53. Geiser C. Data analysis with Mplus. New York: Guilford Press; 2013.

54. Farrell AM. Insufficient discriminant validity: a comment on Bove, Pervan, Beatty, and Shiu (2009). J Bus Res. 2010;63(3):324-7.

55. Li Z, Yin X, Jiang S, Wang M, Cai T. Psychological mechanism of subjective wellbeing: a stable trait or situational variability. Soc Indic Res. 2014;118(2):523-34

56. Lucas RE, Donnellan MB. How stable is happiness? Using the STARTS mode to estimate the stability of life satisfaction. J Res Pers. 2007;41(5):1091-8.

57. Rubenstein CL, Duff J, Prilleltensky I, Jin Y, Dietz S, Myers ND, Prilleltensky O. Demographic group differences in domain-specific well-being. J Community Psychol. 2016:44(4):499-515.

58. Kagan C, Kilroy A. Psychology in the community. In: Haworth J, Hart G, editors. Well-being: individual, community and social perspectives. New York: Palgrave Macmillan; 2007. p. 97-113.

59. Cummins RA. Objective and subjective quality of life: an interactive model. Soc Indic Res. 2000:52(1):55-72.

60. Diener E, Suh E. Measuring quality of life: economic, social, and subjective indicators. Soc Indic Res. 1997:40(1):189-216. 